\title{
Mouse granzyme A induces a novel death with writhing morphology that is mechanistically distinct from granzyme B-induced apoptosis
}

\author{
O Susanto ${ }^{1,2}$, SE Stewart ${ }^{3}$, I Voskoboinik ${ }^{1,2}$, D Brasacchio ${ }^{1,2}$, M Hagn $^{1,2}$, S Ellis ${ }^{4}$, S Asquith ${ }^{4}$, KA Sedelies $^{1,2}$, PI Bird ${ }^{3}$, \\ NJ Waterhouse $\mathrm{s}^{5,6,7,8}$ and JA Trapani ${ }^{*, 1,2,8}$
}

Human and mouse granzyme (Gzm)B both induce target cell apoptosis in concert with pore-forming perforin (Pfp); however the mechanisms by which other Gzms induce non-apoptotic death remain controversial and poorly characterised. We used timelapse microscopy to document, quantitatively and in real time, the death of target cells exposed to primary natural killer (NK) cells from mice deficient in key Gzms. We found that in the vast majority of cases, NK cells from wild-type mice induced classic apoptosis. However, NK cells from syngeneic Gzm B-deficient mice induced a novel form of cell death characterised by slower kinetics and a pronounced, writhing, 'worm-like' morphology. Dying cells initially contracted but did not undergo membrane blebbing, and annexin-V staining was delayed until the onset of secondary necrosis. As it is different from any cell death process previously reported, we tentatively termed this cell death 'athetosis'. Two independent lines of evidence showed this alternate form of death was due to Gzm A: first, cell death was revealed in the absence of Gzm B, but was completely lost when the NK cells were deficient in both Gzm A and B; second, the athetotic morphology was precisely reproduced when recombinant mouse Gzm A was delivered by an otherwise innocuous dose of recombinant Pfp. Gzm A-mediated athetosis did not require caspase activation, early mitochondrial disruption or generation of reactive oxygen species, but did require an intact actin cytoskeleton and was abolished by latrunculin B and mycalolide B. This work defines an authentic role for mouse Gzm A in granule-induced cell death by cytotoxic lymphocytes.

Cell Death and Differentiation (2013) 20, 1183-1193; doi:10.1038/cdd.2013.59; published online 7 June 2013

Cytotoxic lymphocytes (CL) such as cytotoxic T lymphocyte (CTL) and natural killer cells (NK) use granule exocytosis to kill virus-infected and malignant cells. This process involves perforin (Pfp)-mediated delivery of granzymes (Gzms) from $\mathrm{CL}$ to the target cell. The importance of this pathway is evident; Pfp-deficient $\left(\mathrm{Pfp}^{-1-}\right)$ mice succumb to viral infection more readily than wild-type (WT) mice, and spontaneously develop B-cell lymphoma later in life, due to severe defects in NK/CTL cytotoxicity. ${ }^{1,2}$ CLs express multiple Gzms with diverse substrate specificities. Congenital loss of individual Gzms does not result in the severe immunodeficiency of $\mathrm{Pfp}^{-/-}$mice, as CTL/ NKs from Gzm-knockout mice can still induce target cell death, indicating that Gzms display some functional redundancy.

The molecular pathway of Gzm B-mediated apoptosis has been independently elucidated by several groups of investigators, but the contribution of the other Gzms to CL granule-induced death is less clear. Although GzmA was one of the earliest Gzms to be purified and characterised, ${ }^{3}$ its function remains ambiguous. Purified GzmA has been reported to induce target cell death with some features in common with apoptosis, including membrane blebbing, nuclear condensation and early mitochondrial damage. ${ }^{4,5}$ In vitro studies report that GzmA cleaves the mitochondrial protein Ndufs3 within complex I of the electron transport chain, and this is postulated to generate a burst of reactive oxygen species (ROS). ${ }^{5,6}$ This oxidative stress is predicted to cause translocation of the SET complex into the nucleus, where GzmA cleaves components of the SET complex and induces DNA damage through single-stranded nicking, resulting in cell death. ${ }^{5,7}$ However, these findings have been difficult to corroborate and recent studies from several independent groups using recombinant or native protease have reported that GzmA has little cytotoxicity ${ }^{8-10}$ or that GzmA may simply enhance Pfp-mediated membranolysis. ${ }^{8}$ In contrast, studies using $\mathrm{GzmB}^{-1-}$ CTL suggest that GzmA does have a role in target cell death. ${ }^{11,12}$

To address this controversy in a physiologically relevant context, we used intact primary mouse NKs to deliver Gzms in

\footnotetext{
${ }^{1}$ Cancer Cell Death Laboratory, Peter MacCallum Cancer Centre, East Melbourne, Victoria, Australia; ${ }^{2}$ Sir Peter MacCallum Department of Oncology, The University of Melbourne, Parkville, Victoria, Australia; ${ }^{3}$ Department of Biochemistry and Molecular Biology, School of Biomedical Sciences, Monash University, Clayton, Victoria, Australia; ${ }^{4}$ Peter MacCallum Cancer Centre, East Melbourne, Victoria, Australia; ${ }^{5}$ Apoptosis and Cytotoxicity Laboratory, Understanding and Preventing Diseases Theme, Mater Medical Research Institute, South Brisbane, Queensland, Australia; ${ }^{6}$ School of Medicine, University of Queensland, St. Lucia, Queensland, Australia and ${ }^{7}$ Fluorescence Imaging Group, Queensland Institute for Medical Research, Brisbane, Queensland, Australia

*Corresponding author: JA Trapani, Cancer Cell Death Laboratory, Cancer Immunology Program, Peter MacCallum Cancer Centre, Locked Bag 1, A'Beckett Street, Melbourne, Victoria 3002, Australia. Tel: +613 9656 1516; Fax: +613 9656 1414; E-mail: joe.trapani@petermac.org

${ }^{8}$ These authors contributed equally to this work.

Keywords: granzyme; cell death; cytotoxic lymphocyte; natural killer cells; cytoskeleton

Abbreviations: Gzm, granzyme; Pfp, perforin; CL, cytotoxic lymphocyte; CTL, cytotoxic T lymphocyte; NK, natural killer cell; AV, annexin-V; PI, propidium iodide; ROS, reactive oxygen species; MTOC, microtubule organising centre

Received 16.11.12; revised 28.3.13; accepted 30.4.13; Edited by P Vandenabeele; published online 07.6.2013
} 
order to analyse the features of GzmA-mediated cell death. As shown previously, NKs from WT mice induced classic target cell apoptosis. By comparison, $\mathrm{GzmB}^{-1}-\mathrm{NKs}$ induced a slower cell death pathway in which the target cells underwent a set of highly reproducible and distinct morphological changes with a marked delay in phosphatidylserine (PS) externalisation. The phenotype was precisely replicated when recombinant mouse GzmA was delivered with purified Pfp, but lost when NKs lacking both Gzms $A$ and $B$ $\left(\mathrm{GzmA}^{-1-} \mathrm{B}^{-1-}\right)$ were used to kill targets. Utilising timelapse microscopy to characterise hundreds of cell death events, our studies describe for the first time the unique morphology of target cells undergoing GzmA-mediated cell death, and its kinetic and biochemical features at the single cell level.

\section{Results}

GzmB $^{-1-}$ NK cells use GzmA to induce target cell death. The ability of NKs from C57BL/6 WT, or syngeneic Gzm- or Pfp-deficient mice to kill murine MC57 target cells was assessed in a 4-h assay (Figure 1a). The vast majority of NK killing occurred via $\mathrm{Ca}^{2+}$-dependent granule exocytosis, as the addition of EGTA completely blocked cytotoxicity and $\mathrm{Pfp}^{-1-}$ NKs were minimally cytotoxic. GzmB ${ }^{-1-}$ NKs induced $\sim 50 \%$ as much target cell death as WT, whereas $\mathrm{GzmA}^{-1-} \mathrm{B}^{-1-} \mathrm{NKs}$ exhibited virtually none over this timeframe. This strongly suggested that GzmA was the major cause of the residual cytotoxicity of $\mathrm{GzmB}^{-1-} \mathrm{NKs}$.

GzmA induces morphologically distinct cell death. We have previously used timelapse microscopy to distinguish different forms of cell death induced by recombinant granule cytotoxins and intact CL. ${ }^{13}$ We adapted these methods to investigate the morphological and kinetic features of cell death brought about by $\mathrm{GzmB}^{-1-} \mathrm{NKs}$, in comparison with WT, GzmA ${ }^{-1-}$ or GzmA ${ }^{-1-} \mathrm{B}^{-1-}$ NKs. Adherent MC57 target cells undergoing WT NK-induced apoptosis (Figure 1b, Supplementary Movie S1) were easily distinguished from targets undergoing NK-induced lysis (Supplementary Figures S1A and B, Supplementary Movie S2). Apoptotic cells rapidly rounded and detached from the substratum, shrank and underwent intense membrane blebbing, followed by staining with annexin-V (AV)-FITC indicating PS externalisation had occurred (Figure 1bii-iv). Cell death culminated in membrane rupture (secondary necrosis), detected by propidium iodide (PI) uptake (Figure 1bvi). Measuring the relative levels of AV and $\mathrm{PI}$ fluorescence in real time clearly showed that AV binding occurred before PI uptake (Figure 1c). This apoptotic morphology was indistinguishable from that of cells treated with recombinant GzmB delivered by otherwise innocuous ('sublytic') concentrations of Pfp (Supplementary Figures S1C and D). Generally, $<3 \%$ target cells died by NK-mediated
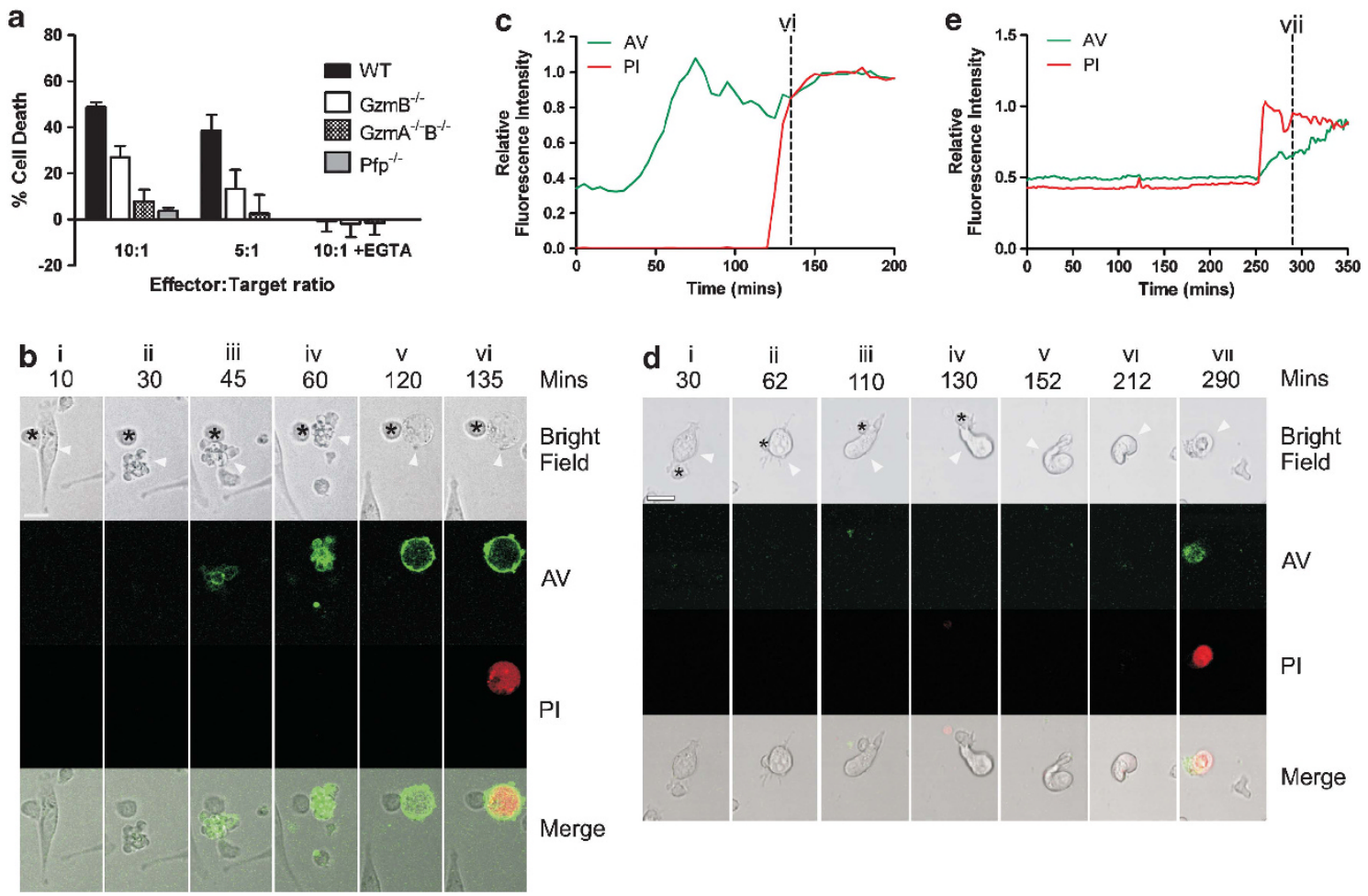

Figure $1 \mathrm{GzmB}{ }^{-1}$ NK cells induce a morphologically distinct form of cell death. (a) ${ }^{51}$ Chromium (Cr)-labelled MC57 target cells incubated for $4 \mathrm{~h}$ with NK cells from WT, $\mathrm{GzmB}^{-1-}, \mathrm{GzmA}^{-1-} \mathrm{B}^{-1-}$ or Pfp ${ }^{-1-}$ mice at the effector:target (E:T) ratios indicated, in the presence or absence of 4 mM EGTA. The level of specific ${ }^{51} \mathrm{Cr}$ release was measured to determine target cell death ( $n=5$, mean \pm S.E.M.). (b-e) Representative timelapse microscopy of MC57 target cells undergoing (b) WT NK-induced apoptosis or (d) a morphologically distinct, alternate form of cell death induced by GzmB ${ }^{-1}$ NKs. Images shown are typical of different stages of NK interaction and target cell death with AV binding and PI uptake. Elapsed time is indicated above each frame. NK cells are indicated by asterisks, target cells by arrowheads, scale bar represents $15 \mu \mathrm{m}$. Relative changes in AV and PI fluorescence over time in the target cells shown in (b) and (d) are graphed in (c) and (e), respectively. The stage representing target cell secondary necrosis (c(vi), e(vii)) is indicated above dotted lines 
lysis, which was morphologically identical to necrosis induced by high concentrations of purified Pfp (Supplementary Figures S1E and F).

In contrast, target cells killed by $\mathrm{GzmB}^{-/}$- NKs displayed a unique morphology distinct from both apoptosis and necrosis. $\mathrm{GzmB}^{-1-}$ NK-killed cells were not tightly rounded, and neither membrane blebbing nor early AV binding were observed (Figures 1d and e). Unlike necrosis, cells did not swell or become rapidly PI permeable. Instead, after gradually rounding and detaching from the substratum (Figure 1dii), target cells became markedly elongated for a prolonged phase, with multiple successive changes in shape reminiscent of a writhing worm-like appearance (Figure 1diii-vi) and culminating in secondary necrosis (Figures 1d and evii, Supplementary Movie S3).

Similar morphology was observed in MS9ll-transformed fibroblasts, B16F10 melanoma cells and YAC-1 lymphoma cells undergoing GzmB ${ }^{-1-}$ NK-mediated death (Supplementary Figures S2A-E). This suggested that this 'alternate' form of death was not peculiar to a certain target cell line; rather, a generic molecular mechanism was likely to underpin the process. Given the striking morphology of GzmA-induced cell death and its resemblance to the slow, writhing movements seen in some neurological disorders, we propose the name athetosis to describe this form of cell death.

When $\mathrm{GzmA}^{-1-} \mathrm{B}^{-1-} \mathrm{NKs}$ were used, this alternate cell death was almost totally abolished in comparison with killing by $\mathrm{GzmB}^{-1-}$ NKs (Figure 2a, Table 1; $P<0.01$ ), confirming that GzmA was indispensable for athetosis. In sharp contrast, both WT and $\mathrm{GzmA}^{-1-}$ NKs induced significantly more apoptosis than either $\mathrm{GzmB}^{-1-}$ or $\mathrm{GzmA}^{-1-} \mathrm{B}^{-1-} \mathrm{NKs}$ $(P<0.0001)$. The fact that athetotic cell death was revealed in the absence of $\mathrm{GzmB}\left(\mathrm{GzmB}^{-1} \mathrm{NKs}\right)$, but lost in $\mathrm{GzmA}^{-1-} \mathrm{B}^{-1-}$ NK-mediated death strongly suggested that
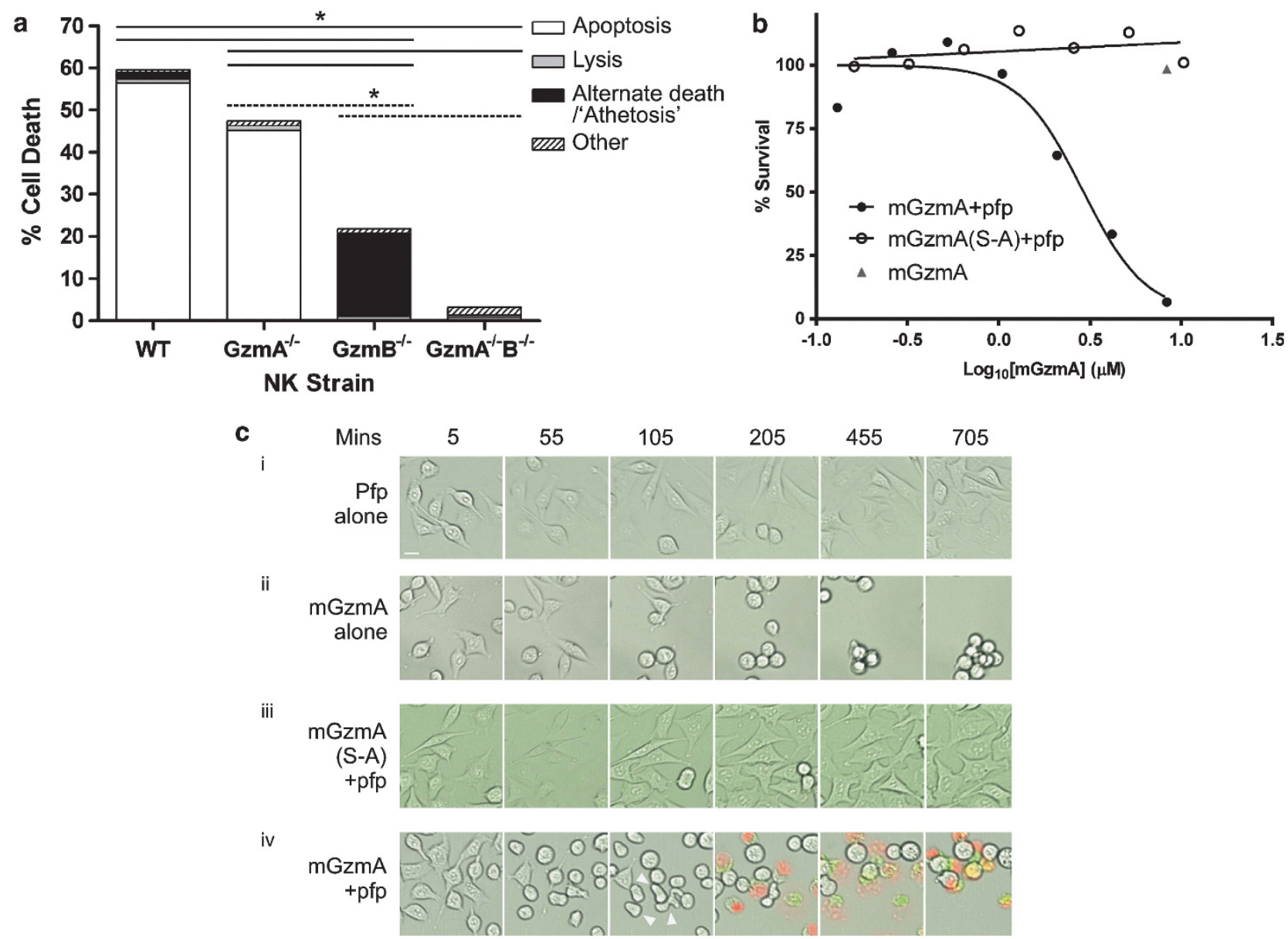

Figure 2 GzmA induces an alternate form of cell death when delivered by NK cells or when delivered as a recombinant protein. (a) The percentage of cells killed by WT, $\mathrm{GzmA}^{-1-}, \mathrm{GzmB}^{-1-}$ or GzmA ${ }^{-1-} \mathrm{B}^{-1-} \mathrm{NK}$ cells, categorised according to morphology of cell death using timelapse microscopy. Each dying cell was classified as either apoptotic, lytic or undergoing alternate death based on the morphological characteristics shown in Figure 1/Supplementary Figure S1; dying cells that could not be classified into any of those categories were noted as 'other'. Three to seven independent experiments were analysed; a total of 335 WT NK-killed cells, 154 GzmA ${ }^{-1}$ - NK-killed cells, $136 \mathrm{GzmB}^{-1-}$ NK-killed cells, $11 \mathrm{GzmA}^{-1-} \mathrm{B}^{-1-}$ NK-killed cells. Statistical analysis was performed using a two-way ANOVA; solid lines indicate comparison of apoptosis, dashed lines indicate comparison of alternate death/athetosis, ${ }^{*} P<0.05$. (b) Cytotoxicity of recombinant $m G z m A$ when delivered into P815 cells with Pfp. Cells were incubated with serial dilutions of wild-type ( $\mathrm{mGzmA}+$ Pfp, closed circles) or inactive mutant ( $\mathrm{mGzmA}$ S-A + Pfp open circles) mGzmA in the presence of Pfp or mGzmA alone in the absence of Pfp (mGzmA, triangle) at the highest dose only $(8 \mu \mathrm{M})$ for $1 \mathrm{~h}$. Cells were allowed to recover overnight then assessed for survival by MTT (3-(4,5dimethylthiazol-2-yl)-2,5-diphenyltetrazolium bromide) assay. $n=3$, representative curve shown. (c) Timelapse microscopy of MC57 target cells treated with $250 \mathrm{nM}$ mGzmA or mGzmA S-A inactive mutant, in the presence or absence of a sublytic dose of Pfp. Images are merged bright field, AV (green fluorescence) and PI (red fluorescence) channels. Arrowheads indicate target cells displaying elongated athetotic morphology. Scale bar: $15 \mu \mathrm{m}$ 
Table 1 Mode of death of MC57 target cells by morphology, induced by NK cells ${ }^{a}$

\begin{tabular}{|c|c|c|c|c|c|}
\hline & Apoptosis & Lysis & Alternate death & Other & Total death \\
\hline WT & $\begin{array}{c}52.56( \pm 9.42) \\
93.11^{\mathrm{b}}\end{array}$ & $\begin{array}{c}0.82( \pm 0.42) \\
1.45\end{array}$ & $\begin{array}{c}1.79( \pm 0.6) \\
3.17\end{array}$ & $\begin{array}{c}1.28( \pm 0.75) \\
2.27\end{array}$ & $\begin{array}{l}56.45 \\
100\end{array}$ \\
\hline GzmA $^{-1-}$ & $\begin{array}{c}45.16( \pm 8.17) \\
95.27\end{array}$ & $\begin{array}{c}1.12( \pm 0.61) \\
2.36\end{array}$ & $\begin{array}{l}0 \\
0\end{array}$ & $\begin{array}{c}1.12( \pm 0.61) \\
2.36\end{array}$ & $\begin{array}{l}47.4 \\
100\end{array}$ \\
\hline $\mathrm{GzmB}^{-1-}$ & $\begin{array}{l}0 \\
0\end{array}$ & $\begin{array}{c}0.98( \pm 0.32) \\
4.3\end{array}$ & $\begin{array}{c}19.77( \pm 3.9) \\
86.67\end{array}$ & $\begin{array}{c}2.06( \pm 0.46) \\
9.03\end{array}$ & $\begin{array}{l}22.81 \\
100\end{array}$ \\
\hline $\mathrm{GzmA}^{-1-} \mathrm{B}^{-1-}$ & $\begin{array}{c}0.66( \pm 0.46) \\
23.15\end{array}$ & $\begin{array}{c}0.66( \pm 0.49) \\
23.15\end{array}$ & $\begin{array}{l}0 \\
0\end{array}$ & $\begin{array}{c}1.53( \pm 0.97) \\
53.7\end{array}$ & $100^{2.85}$ \\
\hline
\end{tabular}

aData corresponds to Figure 2a, the percentage of each type of cell death expressed as mean ( \pm S.E.M.).

${ }^{b}$ Figures in italics are the proportion of each type of cell death, relative to the total amount of cell death induced per NK strain.

(i) the dominant cell death phenotype of WT NKs is GzmBmediated apoptosis and (ii) a second, subdominant pathway mediated by GzmA is revealed when GzmB is removed. We also excluded any role for GzmM in athetosis, using $\mathrm{GzmB}^{-1-} \mathrm{M}^{-1-} \mathrm{NKs}$ (Supplementary Figures S2F-H).

It was important to independently confirm that GzmA alone could bring about the morphology of athetosis. To achieve this, we used purified recombinant Pfp and GzmA. Mouse GzmA ( $m$ GzmA) induced athetosis when applied to mouse P815 cells with sublytic Pfp (Figure 2b). However, target cell death did not occur in the absence of Pfp, or when GzmA with a mutated active-site serine residue ( $\mathrm{mGzmA}$ S-A) was used, indicating that actual cleavage of a key substrate/s was required for cell death. Using timelapse microscopy, we confirmed that treatment of MC57s with sublytic Pfp alone or with mGzmA S-A and Pfp did not induce death or changes in cell morphology (Figure 2c). Treatment with (active) $\mathrm{mGzmA}$ alone resulted in target cell detachment, most likely due to GzmA-mediated cleavage of cell adhesion proteins: ${ }^{14-16}$ however, the cells continued to proliferate and remained viable after $24 \mathrm{~h}$ (Figures $2 \mathrm{~b}$ and $\mathrm{c}$, Supplementary Figure S2I). In contrast, treatment with $\mathrm{mGzmA}$ and Pfp produced the characteristic elongated morphology of $\mathrm{GzmB}^{-1-}$ NK-induced alternate death (Figure 2civ, Supplementary Movie S4). The concordance of the findings using recombinant reagents and intact NK cells provided independent evidence that athetotic cell death is mediated by $\mathrm{mGzmA}$.

The kinetics of GzmA-mediated cell death is slower than GzmB-mediated apoptosis. Despite expressing levels of GzmA equivalent to those in $\mathrm{GzmB}^{-1}$ NKs (Supplementary Figure S3A), WT NKs consistently killed target cells by GzmB-mediated apoptosis (Figure 2a). As athetosis was observed only in the absence of GzmB, we tested the hypothesis that GzmA took longer to initiate cell death compared with GzmB. The length of the initiation phase of cell death (the time from Gzm delivery to the first visible morphological change of cell death, typically cell rounding) was determined using timelapse microscopy. To pinpoint when NK-mediated Gzm delivery had occurred, we used a modified PI uptake assay as a marker of Pfp pore formation in the target cell membrane. Very low concentrations of $\mathrm{PI}$ $(1.0-1.5 \mu \mathrm{M})$ are typically used in cell death assays to detect massive plasma membrane rupture during secondary necrosis. By adding significantly more $\mathrm{PI}(100 \mu \mathrm{M})$ to the medium, PI uptake can be detected entering the target cell specifically at the site of NK synapse upon granule delivery ${ }^{17}$ (Figure 3a). Using these parameters, the initiation phase of GzmB-mediated apoptosis (mediated by WT or GzmA ${ }^{-1-}$ NKs) was $4.9 \pm 0.2$ or $3.3 \pm 0.1 \mathrm{~min}$, respectively, whereas the initiation phase of $\mathrm{GzmB}^{-1}$ - NK-mediated athetosis was significantly longer ( $16.9 \pm 1.02 \mathrm{~min} ; P<0.0001$, Figure $3 b)$. The execution phase of cell death (the length of time from cell rounding to secondary necrosis) was also significantly longer during GzmB ${ }^{-1-}$ NK-mediated death $(P<0.0002)$. Apoptotic cells had an execution phase of $92.2 \pm 5.7$ min (Figure $3 \mathrm{c}$ ), a result similar to that reported previously, ${ }^{13}$ whereas GzmB $^{-1-}$ NK-killed cells took $176.5 \pm 18.5 \mathrm{~min}$ for the equivalent phase. This kinetic data confirmed that GzmB-mediated apoptosis is triggered and executed more rapidly than GzmA-mediated athetosis.

Exploring the mechanism of GzmA-mediated cell death. We wished to address the molecular mechanisms underlying GzmA-induced cell death. Cytochrome $c$ release from mitochondria is a critical early event in human GzmBmediated apoptosis. ${ }^{18}$ To identify a possible role for early cytochrome $c$ release, MC57 target cells were mixed with $\mathrm{GzmB}^{-1-}$ NKs and stained for cytochrome c. Untreated MC57 cells displayed characteristic punctate cytochrome $c$ staining, consistent with mitochondrial localisation (Figure 4a, Supplementary Figure S3B), whereas apoptotic cells treated with recombinant GzmB/Pfp displayed diffuse cytochrome $c$ staining, indicating it had been released into the cytosol. In athetotic cells, cytochrome $c$ staining remained punctate, indicating that early cytochrome $c$ release had not occurred.

We then investigated the requirement for caspase activation. Although WT NK-mediated apoptosis was significantly reduced in the presence of the broad-spectrum caspase inhibitor Q-VD-OPh (QVD; $P<0.05$; Figure 4b), QVD had no significant effect on the amount or morphology of $\mathrm{GzmB}^{-1-}$ NK-mediated cell death (Figures $4 b$ and $c$ ). This confirmed that GzmA-mediated alternate death was caspase independent. It is well known that pro-caspase processing and caspase activation are key to the pro-apoptotic activity of GzmB. ${ }^{18}$ Interestingly, caspase blockade with QVD revealed an increase in the proportion of WT NK-killed cells with athetotic morphology, further indicating that GzmA-induced athetosis can be 'masked' by GzmB (Supplementary Figure $\mathrm{S} 3 \mathrm{C}$ ). However, this athetotic morphology was accompanied by some residual membrane blebbing, most likely due to GzmB directly inducing blebbing without caspase activation. ${ }^{19}$ 
a

Mins

i

ii

iii

iv

77

WT
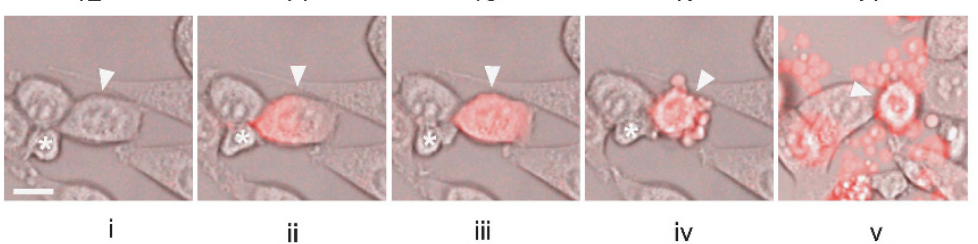

$7 \quad$ ii

iii

iv

30

$\mathrm{GzmA}^{*}$
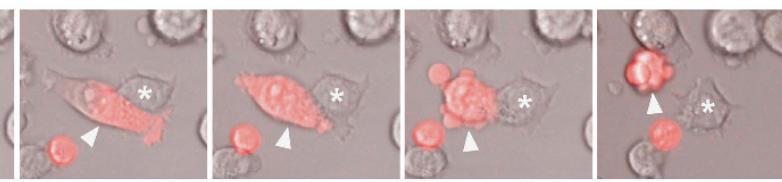

i
27

ii

iii

iv
57

185

$\mathrm{GzmB}^{+}$

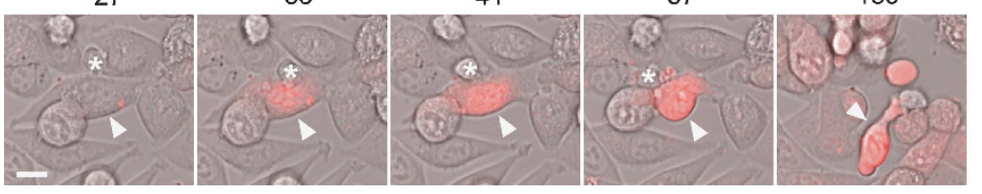

c

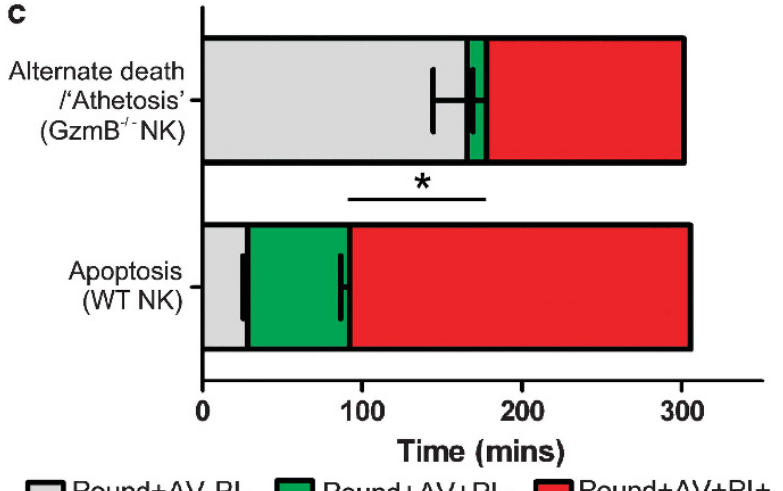

Figure 3 Induction of GzmB ${ }^{-1-}$ NK-mediated alternate cell death is initiated at a slower rate to WT NK-mediated apoptosis. (a) Timelapse microscopy of MC57 targets killed by WT, GzmA ${ }^{-1-}$ or GzmB ${ }^{-1-}$ NK cells, with $100 \mu \mathrm{M}$ PI present in the culture medium. Representative images of different stages of NK killing and cell death are shown. (ii) NK-mediated granule delivery and Pfp polymerisation is detected by PI uptake in the target. (iv) Target cell rounding was indicated by cell contraction and/or increased contrast around the cell. Asterisks indicate NK cells, arrowheads indicate target cells. Scale bar: $10 \mu \mathrm{m}$. (b) The kinetics of the initiation phase of death - the elapsed time from NK hit (PI uptake) to cell rounding. Each individual NK/target interaction is represented by a single point, the mean is indicated by the black bars. $n=6$ ( 285 WT NK-killed cells, $208 \mathrm{GzmA}^{-1-}$ NK-killed cells and $158 \mathrm{GzmB}^{-1}$ - NK-killed cells analysed). Statistical analysis was performed using a one-way ANOVA, * $P<0.0001$. (c) The kinetics of the execution phase of cell death determined using timelapse microscopy, with AV and $1.5 \mu \mathrm{M} \mathrm{PI}$ in the culture medium. The mean ( \pm S.E.M.) length of time from cell rounding (grey bar) to AV binding (green bar) and PI uptake/secondary necrosis (red bar) is shown. $n=9$ (147 WT NK-killed cells and 82 GzmB ${ }^{-1-}$ NK-killed cells). Statistical analysis was performed using a Mann-Whitney $U$ test, ${ }^{*} P<0.0002$

Although morphologically and kinetically distinct from necrosis, we also tested whether athetosis may converge on the programmed necrosis/necroptosis pathway, which requires RIP1. ${ }^{20}$ However, treatment with the RIP1 inhibitor necrostatin-1 did not inhibit $\mathrm{GzmB}^{-1-}$ NK cell-mediated death, either in the presence or absence of QVD (Supplementary Figure S3D).

Early mitochondrial damage that induces increased ROS and loss of mitochondrial transmembrane potential $\left(\Delta \Psi_{\mathrm{m}}\right)$ is considered a critical event for GzmA-induced cell death, ${ }^{4,5,12}$ and the presence of superoxide scavengers such as tiron can significantly inhibit GzmA-induced cell death. ${ }^{5,6}$ The fluorescent dye tetramethylrhodamine ethyl ester (TMRE) was used with timelapse microscopy to assess whether loss of $\Delta \Psi_{\mathrm{m}}$ is an early feature of $\mathrm{GzmB}^{-1-}$ NK-mediated death. MC57 cells treated with the mitochondrial uncoupler FCCP and HeLa cells undergoing apoptosis imposed by the human NK cell line KHYG1 showed significant loss of TMRE fluorescence before cell rounding, in comparison with untreated cells $(P<0.005$; Figure $4 \mathrm{~d}$, Supplementary Figures S4A and B). In contrast, there was no change in TMRE fluorescence in $\mathrm{GzmB}^{-1-}$ NK-killed cells before cell rounding (Figure $4 d$, Supplementary Figures $\mathrm{S} 4 \mathrm{C}$ and $\mathrm{D}$ ), demonstrating that early loss of $\Delta \Psi_{\mathrm{m}}$ is not necessary for GzmA-mediated athetosis. Next, we used the ROS-sensitive fluorescent dye dihydroethidium (DHE) to detect early increased intracellular ROS. As GzmA-mediated ROS production was thought to occur following disruption of mitochondrial complex I, we used the complex I inhibitor rotenone to demonstrate induction of ROS production (Figure 4e). In contrast, neither WT 
a

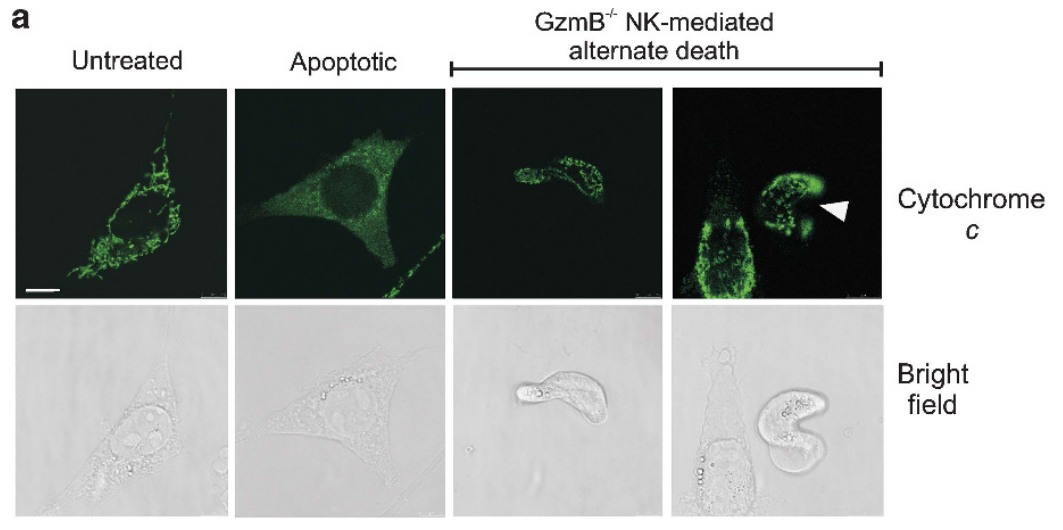

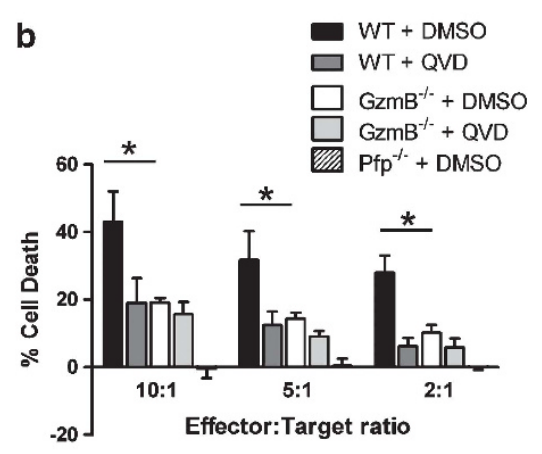

C Mins 55

85

145

165

195

250

DMSO

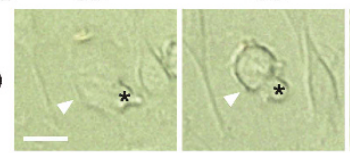

140

155
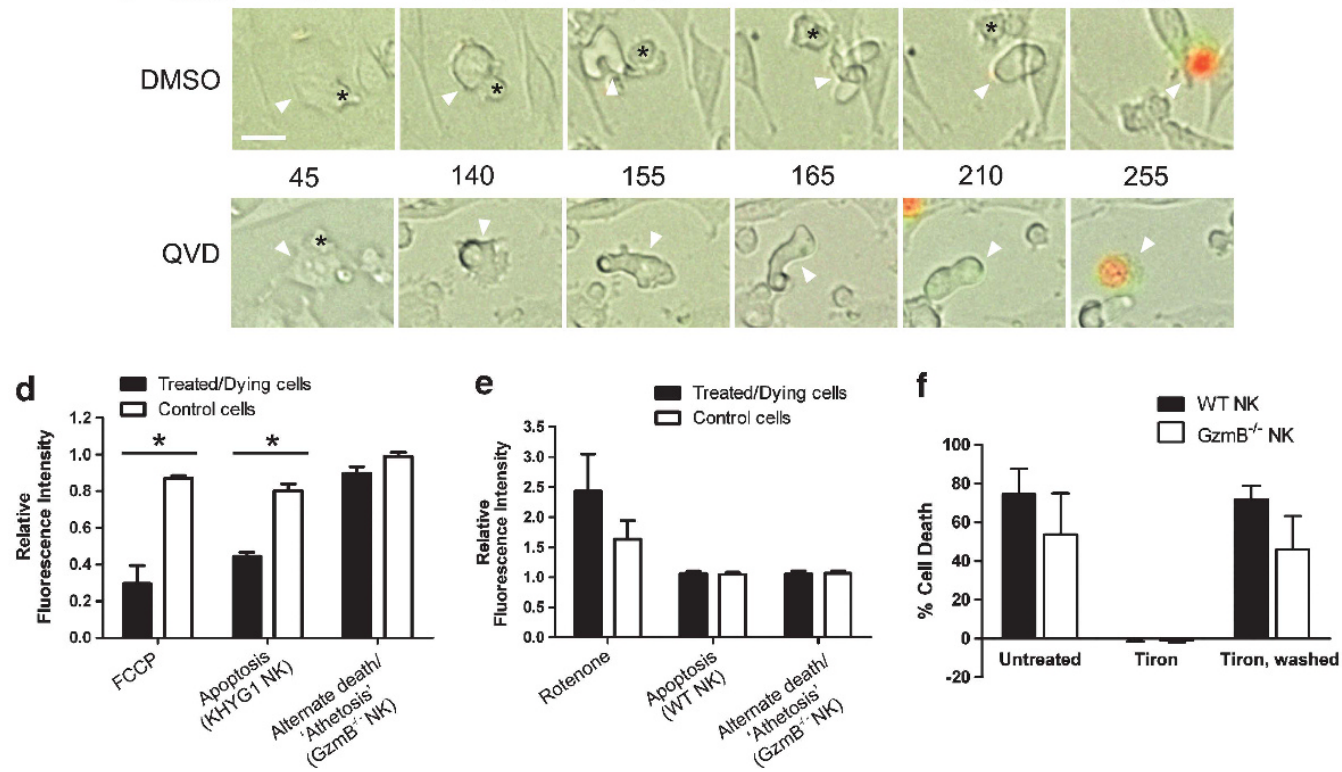

Figure 4 Alternate cell death induced by GzmB ${ }^{-1}$ NK cells does not display features of apoptosis or early mitochondrial damage. (a) Cells undergoing GzmA-mediated alternate death do not display early cytochrome $c$ release. MC57 cells were treated with human recombinant GzmB + Pfp for 10 min to induce apoptotic cytochrome $c$ release or mixed with $\mathrm{GzmB}^{-1-}$ NK cells for $2 \mathrm{~h}$ until morphological changes were apparent, then fixed and stained for cytochrome $c$. Representative images of four independent experiments are shown. Scale bar: $10 \mu \mathrm{m}$. (b) GzmB ${ }^{-1-}$ NK cell-induced alternate death is independent of caspase activation. ${ }^{51} \mathrm{Cr}$-labelled MS9ll target cells were incubated for $4 \mathrm{~h}$ with WT or GzmB ${ }^{-1}$ NK cells in the presence of either DMSO or $40 \mu \mathrm{M} \mathrm{QVD}$. Statistical analysis was performed using a two-way ANOVA, ${ }^{*} P<0.05(n=3$, mean \pm S.E.M.). (c) The morphology of GzmA-mediated alternate death occurs independently of caspase activation. Timelapse microscopy of MS9ll target cells killed by $\mathrm{GzmB}^{-1-}$ NK in the presence of either DMSO (top row) or $40 \mu \mathrm{M}$ QVD (bottom row). Images are merged bright field, green (AV) and red (PI) fluorescence channels. Target cells are indicated with arrowheads, NK cells by with asterisks. Scale bar: $10 \mu \mathrm{m}$. (d) The overall relative change in TMRE fluorescence in target cells during the initiation phase of cell death. MC57 cells were loaded with TMRE, treated with $33 \mu \mathrm{M} \mathrm{FCCP} \mathrm{or} \mathrm{mixed} \mathrm{with} \mathrm{GzmB}{ }^{-1}$ - NK cells to induce alternate cell death, while HeLa targets were loaded with TMRE and mixed with KHYG1 NK cells to induce apoptosis via the intrinsic pathway (black bars). Cells that were untreated, or did not undergo NK attack show background levels of change in fluorescence (white bars). $n=3-5$ (32 FCCP-treated cells, 37 KHYG1-killed cells, $31 \mathrm{GzmB}^{-1-}$ NK-killed cells). Statistical analysis was performed using an unpaired $t$-test, ${ }^{*} P<0.05$ (mean \pm S.E.M.). (e) The overall relative change in DHE fluorescence in target cells during the initiation phase. MC57 cells were loaded with DHE and treated with $0.4 \mathrm{mM}$ rotenone or mixed with WT or $\mathrm{GzmB}^{-1-}$ NK cells to induce apoptosis or alternate cell death, respectively (black bars), in comparison with control cells (white bars). $n=3-9$ (22 rotenone-treated cells, 41 WT NK-killed cells and 41 GzmB ${ }^{-l}$ - NK-killed cells). (f) Tiron blocks NK killing when present in the culture medium. ${ }^{51} \mathrm{Cr}$-labelled MC57 target cells were either preincubated for $30 \mathrm{~min}$ with $80 \mathrm{mM}$ tiron, and then washed or left untreated, and mixed with WT or GzmB ${ }^{-1}$

NK cells at a 10:1 E:T ratio for 4 h. $n=2$ (mean \pm S.E.M.)

NK-killed apoptotic cells nor GzmB ${ }^{-1-}$ NK-killed athetotic cells displayed detectable ROS before cell rounding (Figure 4e, Supplementary Figures S4E-H). Finding these results inconsistent with previous reports, we also tested the effect of tiron on WT and $\mathrm{GzmB}^{-1}$ NK-mediated death. Although the continual presence of tiron did inhibit all target cell death, death was not inhibited if the targets were washed after preincubation with tiron (Figure 4f). This suggested that tiron was exerting its effect extracellularly rather than by blocking ROS intracellularly. We subsequently found that tiron is a highly effective inhibitor of recombinant Pfp when used at these concentrations (Supplementary Figure S5). This may be a more feasible explanation of the inhibitory effects of tiron on intact NKs (especially WT NKs) and following GzmA/Pfp treatment, rather than by acting as a superoxide scavenger. 
GzmA-mediated athetosis requires a functional actin cytoskeleton. The distinctive morphology of target cells undergoing athetosis prompted us to determine whether perturbation of the cell cytoskeleton was involved. Both actin and tubulin were previously identified as potential targets of GzmA; ${ }^{21}$ thus, we performed immunofluorescence staining for polymerised actin, $\alpha$-tubulin and $\gamma$-tubulin. In comparison with healthy, adherent target cells, athetotic cells appeared to have stronger cortical, and more diffuse cytoplasmic phalloidin staining (Figure 5aii and iii, Supplementary Figures S6A and B) while $\alpha$-tubulin staining was less well defined. However, cells undergoing apoptosis displayed similar staining (Figure 5ai), suggesting that the changes may reflect cell detachment to some extent or be a generic effect of cell death. There was no evidence for redistribution of polymerised actin or $\alpha$-tubulin. However, $\gamma$-tubulin staining in these cells was altered in comparison with untreated cells
(Figure 5b). $\gamma$-tubulin is important for microtubule nucleation and accumulates at the centrosome with the microtubule organising centre (MTOC) ${ }^{22}$ In almost all healthy adherent cells, $\gamma$-tubulin staining was concentrated at one to two well-defined points adjacent to the nucleus, consistent with centrosomal/MTOC localisation ${ }^{23}$ (Supplementary Figure $\mathrm{S} 6 \mathrm{Ci})$. The majority of apoptotic cells $(70.9 \pm 10.9 \%)$ also had normal $\gamma$-tubulin localisation (Supplementary Figure S6Cii). Conversely, a high proportion of athetotic cells displayed significant MTOC disruption (Figure 5b): $50.9 \pm 8.8 \%$ lacked an observable MTOC and $27 \pm 7.3 \%$ of cells showed MTOC repositioning away from the nucleus (Supplementary Figure S6Civ-v). To test whether functional microtubules are required for GzmA-induced death, we pretreated target cells with taxol (Figure $5 \mathrm{c}$ ) or nocodazole (Supplementary Figure S6D), which respectively stabilise or disrupt microtubules; however, neither agent had a
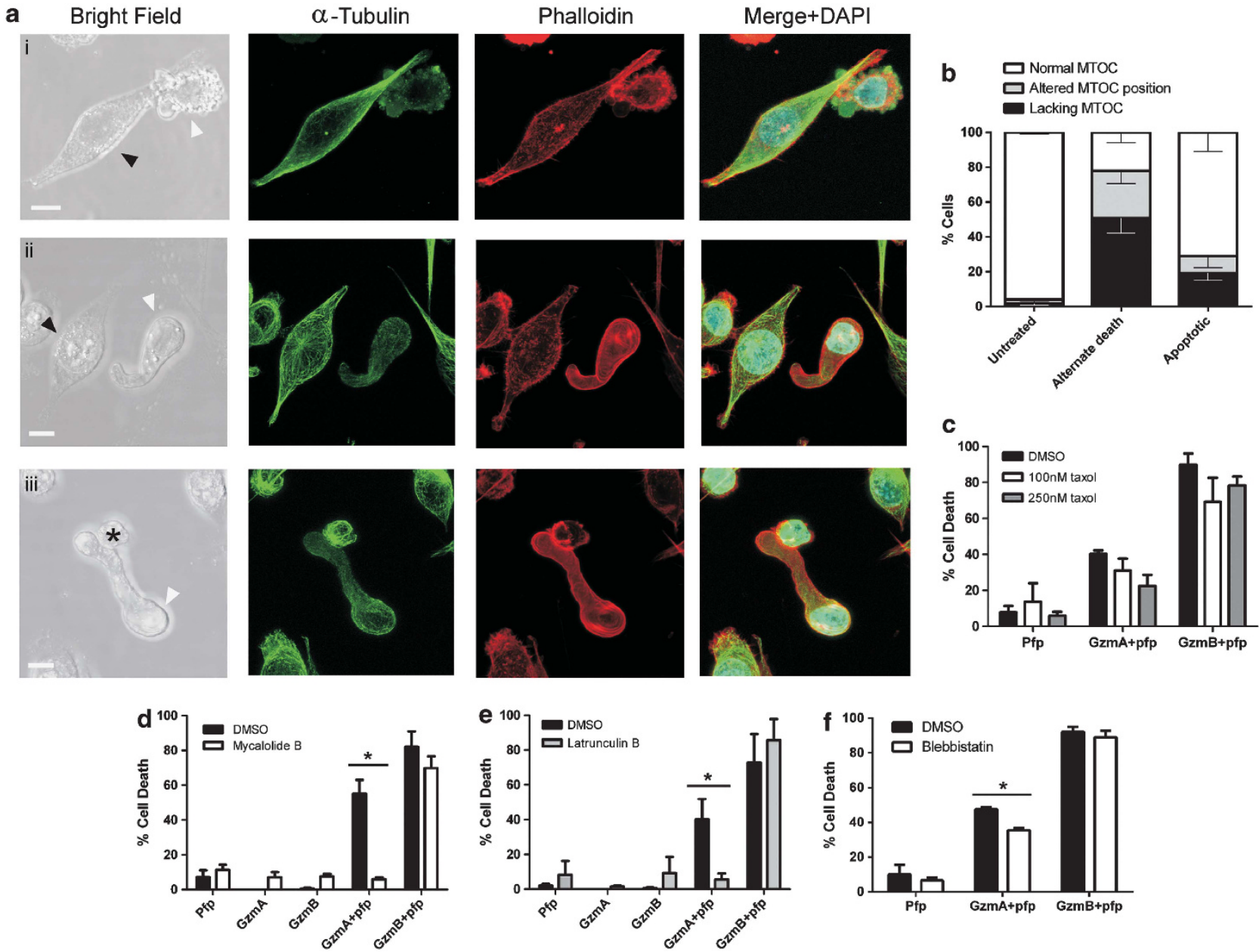

Figure 5 Induction of GzmA-mediated alternate death requires the presence of a functional actin cytoskeleton. (a) MC57 target cells stained with phalloidin-TRICT, anti- $\alpha$ tubulin and DAPI (max z-projections shown), undergoing either (i) WT NK-mediated apoptosis (white arrowhead) or (ii, iii) GzmB ${ }^{-1}$ - NK-mediated alternate death (white arrowhead) and healthy adherent cells in the same fields (black arrowheads), NK cells are indicated by asterisks. Scale bar: $7.5 \mu \mathrm{m}$. (b) MC57 cells mixed with either WT or $\mathrm{GzmB}^{-1-}$ NKs were stained with anti- $\gamma$-tubulin and DAPI, and analysed by confocal microscopy for $\gamma$-tubulin/MTOC localisation, and then classified for alterations in MTOC localisation as described in Supplementary Figure S6C. Mean \pm S.E.M. of $n=4$ (560 untreated targets, 103 GzmB ${ }^{-1-}$ NK-killed and 119 WT NK-killed target cells). (c-f) MC57 target cells pretreated with DMSO, (c) taxol, (d) $2 \mu \mathrm{M} \mathrm{MycB,} \mathrm{(e)} 10 \mu \mathrm{M}$ LatB or (f) $10 \mu \mathrm{M}$ blebbistatin were treated with $250 \mathrm{nM}$ recombinant mGzmA or $120 \mathrm{nM}$ recombinant human GzmB in the presence or absence of sublytic Pfp. Target cell death over $12 \mathrm{~h}$ was assessed via timelapse microscopy and quantified. Data was pooled from $(\mathbf{c}, \mathbf{e}, \mathbf{f})$ three or (d) six independent experiments. Statistical analysis was performed using an unpaired $t$-test, ${ }^{*} P<0.05$ (mean \pm S.E.M.) 
significant effect on GzmA-mediated athetosis. By contrast, we found that GzmA-mediated cell death was almost totally abolished when actin polymerisation was inhibited in target cells, by pretreatment with either mycalolide B (MycB; $P<0.001$; Figure 5d) or latrunculin B (LatB; $P<0.05$; Figure $5 e$ ), yet these agents had no effect on GzmB-mediated death. In addition, inhibition of the actin-associated motor protein myosin II with the small molecule blebbistatin produced a minor reduction in GzmA-mediated death (Figure 5f). Although statistically significant, this reduction in cell death $(\sim 20 \%)$ was far less marked than with MycB/LatB (>80\%). Collectively, these results point to an intact actin cytoskeleton being required for GzmA-mediated athetosis.

\section{Discussion}

The cytotoxicity of GzmA has become subject to much debate, with various studies suggesting that GzmA induces target cell death weakly (or not at all), and is more important for mediating a proinflammatory signal. ${ }^{8-10,24}$ Here, we have demonstrated that cell death can be induced when mGzmA is delivered by NKs via authentic CL/target cell interactions.

Our study also shows for the first time that GzmA-mediated death is morphologically distinct from apoptosis and necrosis, and brings about striking cell elongation and a worm-like morphology in the dying cell before secondary necrosis. Critical for validating GzmA's role, the alternative form of cell death was induced by intact $\mathrm{GzmB}^{-/}$NKs but completely lost when using $\mathrm{GzmA}^{-/-} \mathrm{B}^{-1-} \mathrm{NKs}$, and recapitulated by delivering purified recombinant $m$ GzmA to target cells with Pfp. Using this distinct morphology to specifically identify cells undergoing GzmA-mediated death, we analysed hundreds of individual cellular deaths to clarify the molecular mechanisms that were consistently and authentically associated with it. Our finding that GzmA induces a caspase-independent form of death without cytochrome $c$ release agrees with previous findings, ${ }^{4,5,12}$ but our finding that it did not involve membrane blebbing, early $\Delta \Psi_{\mathrm{m}}$ loss or early ROS production contrasts with others. ${ }^{4,5}$ The absence of mitochondrial damage during the early stages of $\mathrm{GzmB}^{-1-} \mathrm{NK}$-mediated cell death was unexpected, given its prominent role in initiating the GzmA cell death pathway. ${ }^{5}$ However, the extensive use of timelapse microscopy and our quantitative analyses allowed us to ascertain events occurring during the initiation phase of death with a high degree of certainty and reproducibility, discriminating them from similar events occurring as a consequence of cell death during the execution phase. We also found major drawbacks in using tiron as a superoxide scavenger in assays requiring $\mathrm{Pfp}$, as the concentrations of tiron previously utilised $^{5}$ caused severe disruption of Pfp function. Downstream of ROS production, cleavage of the SET complex was reported to be another critical event in GzmA-mediated death. ${ }^{5,25}$ Given the lack of ROS generation as a trigger, it is unclear whether mGzmA would induce SET cleavage in vivo; however, we found that target cell treatment with recombinant $m G z m A$ or GzmB with Pfp did induce cleavage of the SET complex component Ape-1 in vitro (data not shown). This correlates with reports that WT, $\mathrm{GzmA}^{-1-}$, $\mathrm{GzmB}^{-1-}$ and $\mathrm{GzmA}^{-1-} \mathrm{B}^{-1-} \mathrm{CTL}$ were equally able to induce Ape-1 cleavage in target cells, ${ }^{12}$ suggesting that SET complex cleavage may not always be a GzmA-specific event.

Our data indicates that any mitochondrial dysfunction is more likely to be a late consequence of GzmA-induced death, occurring downstream of the cytoskeletal alterations associated with cell rounding/detachment and the worm-like morphology. Indeed, it has been previously hypothesised that GzmA may induce actin cytoskeletal disruption, resulting in downstream ROS generation similar to apoptosis in yeast. $^{12,26}$ Here, we have reported the critical and novel finding that an intact actin cytoskeleton is crucial for GzmAmediated athetosis, but is not required for GzmB-mediated apoptosis. Inhibition of myosin II also caused a mild, although statistically significant reduction in GzmA-mediated death. Given the role of myosin II in regulating actin dynamics in migrating cells, ${ }^{27}$ we hypothesise that myosin may also influence the function of the actin network during athetosis. We also discovered that a high proportion of cells undergoing GzmA-mediated alternate death had altered MTOC localisation; however, microtubule-disrupting agents had no effect, indicating that the actin cytoskeleton has the more critical role. Future studies will aim to identify GzmA substrates linked to the actin cytoskeleton to unravel the precise role of the cytoskeleton in athetosis.

Although it has often been speculated that GzmA has a 'backup' role in CL-mediated cytotoxicity, given the dominance of GzmB-mediated apoptosis, until now there has been no way to formally demonstrate this point using intact $\mathrm{CL}$ containing both GzmA and GzmB. The identification of a unique morphology for GzmA-mediated death has been critical in showing that WT NKs can induce both GzmBmediated apoptosis and GzmA-mediated athetosis; however, apoptosis is far more commonly induced. The dominance of apoptosis may be partly explained by the significantly shorter initiation phase of apoptosis in comparison with athetosis, most likely due to the rapid activation of the caspases. However, in vivo this disparity in time would make very little difference to overall NK cytotoxicity. Although the morphology of WT NK-induced cell death is more akin to 'classic' apoptosis, in reality a 'hybrid' form of death involving nearsimultaneous cleavage of substrates by both GzmA and GzmB may be taking place in the target cell, with GzmAmediated cytoskeletal changes contributing to death of the cell and its disassembly, and GzmB activating the caspases and producing the dominant phenotype.

Although GzmA-mediated cell death may also be an important fallback mechanism if apoptosis were blocked, we have consistently found $\mathrm{GzmB}^{-1-} \mathrm{NKs}$ to be less cytotoxic than WT NKs, and that recombinant GzmB induces significantly more death than the equivalent concentration of GzmA, consistent with previous reports. ${ }^{10,28}$ This suggests that GzmA cannot fully compensate for the absence of GzmB. Thus, the data presented in this paper, in conjunction with previous findings, ${ }^{13}$ reinforces the hypothesis that although delivered simultaneously, Gzms function in a hierarchy during CL-mediated cell death (Table 2). Foremost is GzmB, which induces cell death most rapidly via apoptosis. GzmA provides a second line of attack, acting via non-apoptotic pathways that are somewhat slower to be initiated and completed, and can be masked by GzmB. Lastly, the other 'orphan' Gzms may be 
Table 2 Hierarchy of granzyme-mediated death in cytotoxic lymphocytes

\begin{tabular}{|c|c|c|c|c|c|c|}
\hline & Cytotoxicity & Morphology & AV & PI & $\begin{array}{c}\text { Initiation } \\
\text { phase (min) }\end{array}$ & $\begin{array}{l}\text { Execution } \\
\text { phase (min) }\end{array}$ \\
\hline $\begin{array}{l}\text { WT NK (GzmB/ } \\
\text { GzmA simultaneous } \\
\text { 'hybrid death' - } \\
\text { GzmB dominant) }\end{array}$ & +++++ & $\begin{array}{l}\text { Apoptotic } \\
\text { (condensed } \\
\text { cell, blebbing) }\end{array}$ & Early & $\begin{array}{l}\text { Late (upon } \\
\text { secondary necrosis) }\end{array}$ & $4.9( \pm 0.2)$ & $92.2( \pm 5.7)$ \\
\hline $\begin{array}{l}\mathrm{GzmA}^{-1-} \mathrm{NK} \\
\text { (GzmB dominant) }\end{array}$ & +++++ & $\begin{array}{l}\text { Apoptotic } \\
\text { (condensed } \\
\text { cell, blebbing) }\end{array}$ & Early & $\begin{array}{l}\text { Late (upon } \\
\text { secondary necrosis) }\end{array}$ & $3.3( \pm 0.1)$ & Unknown \\
\hline $\begin{array}{l}\text { GzmB }-1-N K \\
\text { (GzmA dominant) }\end{array}$ & +++ & $\begin{array}{l}\text { 'Worm-like' } \\
\text { (elongated cell, } \\
\text { no blebbing) }\end{array}$ & \multicolumn{2}{|c|}{ Simultaneous (upon secondary necrosis) } & $16.9( \pm 1.02)$ & $176.5( \pm 18.5)$ \\
\hline $\begin{array}{l}\text { GzmA }^{-1-} \mathrm{B}^{-1-} \\
\text { CTLa } / \mathrm{NK} \\
\text { (other Gzms) }\end{array}$ & + & $\begin{array}{l}\text { Similar to } \\
\text { apoptotic } \\
\text { (blebbing) }^{\mathrm{a}}\end{array}$ & \multicolumn{2}{|c|}{ Simultaneous (upon secondary necrosis) ${ }^{a}$} & Unknown & $87.4( \pm 3.2)^{a}$ \\
\hline
\end{tabular}

${ }^{\mathrm{a} D a t a}$ from $\mathrm{GzmA}^{-1-} \mathrm{B}^{-1-} \mathrm{CTL}$ as previously described. ${ }^{13}$

deployed if the principal Gzms are blocked, presumably using different pathways to bring about cell death.

In a pathophysiological context, the clearest example of reliance on multiple Gzms for an organism's survival is mouse infection with the natural poxvirus ectromelia. $\mathrm{GzmA}^{-/-}$and $\mathrm{GzmB}^{-1-}$ mice each showed only marginally increased susceptibility to ectromelia in comparison with WT; however, $\mathrm{GzmA}^{-1-} \mathrm{B}^{-1-}$ mice were highly susceptible, similar to $\mathrm{Pfp}^{-1-}$ mice. ${ }^{29}$ This demonstrates the importance of different Gzms cooperatively mediating alternative pathways to cell death. Consistent with previous reports showing modest cytotoxic activity for $\mathrm{mGzmA}$, but none for its human counterpart, ${ }^{10}$ we found that proteolytically active recombinant human GzmA (hGzmA) was very poorly cytotoxic when administered with Pfp (Supplementary Figure S7). As hGzmA has been hypothesised to be more involved in promoting proinflammatory immune responses rather than direct cytotoxicity, ${ }^{8}$ our data supports the possibility that hGzmA may have developed into a primarily non-cytotoxic immune regulator, whereas $\mathrm{mGzmA}$ retained its cytotoxicity, possibly in response to various mouse pathogens.

\section{Materials and Methods}

Cell culture and mice. Mouse MC57 (fibrosarcoma) cells were maintained in DMEM (Invitrogen Life Technologies, Carlsbad, CA, USA) containing $2 \mathrm{mM}$ L-glutamine and $10 \% \mathrm{FCS}$, at $37^{\circ} \mathrm{C}$ with $10 \% \mathrm{CO}_{2}$. The human cell line HeLa was maintained as previously described. ${ }^{30}$

Primary mouse NK cells were isolated and purified from mouse by negative selection (Mouse NK Isolation Kit, MACS, Miltenyi Biotec GmbH, Bergisch Gladbach, Germany) as previously described, ${ }^{31}$ and cultured in RPMI 1640 media supplemented as above with 1000 units $/ \mathrm{ml}$ human recombinant IL-2. NKs were used for cytotoxicity assays between day 5 and 8 following isolation.

C57BL/6 WT mice were bred at the Walter and Eliza Hall Medical Research Institute and maintained at the Peter MacCallum Cancer Centre. GzmA ${ }^{-1-32}$ $\mathrm{GzmB}^{-1-33} \mathrm{GzmA}^{-1-} \mathrm{B}^{-1-34}$ and $\mathrm{Pfp}^{-1-35}$ mice were generated as previously described and maintained at the Peter MacCallum Cancer Centre. All mice were maintained under SPF conditions.

Antibodies and reagents. The following were used for confocal microscopy: mouse monoclonal anti-cytochrome $c(1: 500$, clone 6H2.B4, BD Pharmingen, San Diego, CA, USA), mouse monoclonal anti- $\alpha$ tubulin $(1: 1000$, Sigma Aldrich, St. Louis, MO, USA), rabbit polyclonal anti- $\gamma$ tubulin (1:1000, Abcam, Cambridge, UK), phalloidin and DAPI (Sigma Aldrich). Secondary antibodies used to detect the primary antibodies were AlexaFluor 488-conjugated goat anti-mouse $\operatorname{lgG}$ and AlexaFluor 546-conjugated goat anti-rabbit IgG (Invitrogen Life Technologies). Other reagents used were FCCP (Invitrogen Life Technologies), Q-VD-OPh (Merck Millipore, Darmstadt, Germany), rotenone (Invitrogen Life Technologies), tiron (Sigma Aldrich), MycB (Enzo Life Sciences, Farmingdale, NY, USA), LatB (Sigma Aldrich), taxol (Santa Cruz Biotechnology, Santa Cruz, CA, USA) and blebbistatin (active enantiomer, Sigma Aldrich).

Recombinant human GzmB, hGzmA and mouse GzmA were produced as previously described. ${ }^{36}$ Recombinant mouse GzmA (mGzmA) WT and active-site mutant were produced by amplifying the $\mathrm{mGzmA}$ coding sequence from a plasmid template by PCR using primers that replaced the signal sequence with a combined FLAG tag and enterokinase cleavage site at the $\mathrm{N}$-terminus and added hexahistidine tag at the C-terminus. The product was the cloned into pPIC9, transformed into Pichia. pastoris and analysed as previously described. ${ }^{36}$ For protein production, $P$. pastoris expressing $\mathrm{mGzmA}$ were grown at $30^{\circ} \mathrm{C}$ for $36 \mathrm{~h}$, and then allowed to settle for $12 \mathrm{~h}$ at room temperature; growth medium was replaced with induction medium containing $3 \%$ methanol and $0.5 \mathrm{M}$ arginine. Cells were induced at $23^{\circ} \mathrm{C}$ for $60 \mathrm{~h}$ and again allowed to settle for $12 \mathrm{~h}$ at room temperature. $\mathrm{mGzmA}$ was then purified from induction medium as previously described, ${ }^{36}$ except at pH 8.0. Once activated, mGzmA was separated from the enterokinase with a further SP-sepharose column, the final activated $\mathrm{mGzmA}$ was concentrated and stored at $-70^{\circ} \mathrm{C}$. SDS-PAGE analysis $+/$ - DTT confirmed that $>99 \%$ of recombinant $m G z m A$ was dimeric. Activity assessed by binding a serpin indicates that $>95 \%$ of the $\mathrm{mGrA}$ was enzymatically active.

Cytotoxicity assays. Pfp, Gzm and NK cell cytotoxicity was assessed by ${ }^{51}$ Chromium-release assays, sheep red blood cell lysis assays or 3-(4,5dimethylthiazol-2-yl)-2,5-diphenyltetrazolium bromide assay, as previously described. ${ }^{37-40}$ To assess cell death by timelapse microscopy, target cells were plated in 96-well flat bottom cell culture plates (Grenier Bio-One GmbH, Frickenhausen, Germany) at a density of $0.5 \times 10^{4}$ cells per well and incubated overnight under standard tissue culture conditions. In some experiments, cells were pretreated for $24 \mathrm{~h}$ with $100 / 250 \mathrm{nM}$ taxol, or for $1 \mathrm{~h}$ with $2 \mu \mathrm{M} \mathrm{MycB}, 10 \mu \mathrm{M}$ LatB, $5 \mu \mathrm{M}$ nocodazole or $10 \mu \mathrm{M}$ blebbistatin. Before induction of cell death, $\mathrm{PI}$ $(1.5 \mu \mathrm{M}$ final) and AV-FITC $(2 \mu \mathrm{g} / \mathrm{ml}$ final, BD Biosciences, San Jose, CA, USA) were added. Cell death was induced by either adding NK cells or by recombinant Gzm/Pfp treatment. Before recombinant Gzm/Pfp treatment, cells were washed three times in DMEM media containing 0.5\% BSA (Fraction V, Roche Diagnostics $\mathrm{GmbH}$, Basel, Switzerland). Recombinant human GzmB, hGzmA or mGzmA were diluted in DMEM/BSA buffer and added to target cells before addition of a sublytic dose of recombinant mouse Pfp (purified in house to a concentration of $300 \mu \mathrm{g} / \mathrm{ml}$, as previously described ${ }^{41}$ ).

Cells were imaged every 5 or 10 min using a $10 \times 10.3$ NA air objective on an Olympus IX-81 microscope (Olympus, Tokyo, Japan) with a temperature-controlled stage (Prior ProScan; GT Vision, Suffolk, UK) and CCD camera (model ORCA-ER; Hamamatsu Photonics KK, Hamamatsu City, Japan). The atmosphere was enriched with $\mathrm{CO}_{2}$ controlled with an active gas mixer (Temperature/ $\mathrm{CO}_{2}$ Environment chamber; Solent Scientific, Segensworth, UK). Laser lines (488 and $561 \mathrm{~nm}$ ) were used to detect AV and PI, respectively. In each experiment, two 
separate fields of view were imaged per condition and 50-200 cells per field were randomly selected. The proportion of cells undergoing cell death was calculated as the number of target cells, which became PI positive.

Confocal timelapse microscopy. Confocal timelapse microscopy to assess intracellular fluorescence was performed with a Leica TCS SP5 spectral confocal microscope system (Leica Microsystems GmbH, Wetzlar, Germany) and an active gas mixer (The Brick; Life Imaging Services, Basel, Switzerland). Adherent target cells were plated in ibiTreat eight-well chamber slides (ibidi GmbH, Martinsried, Germany) at a density of $1.5-3 \times 10^{4}$ cells per well. The confocal pinhole was opened up to a minimum of $150 \mu \mathrm{m}$ wide to allow fluorescence from a thicker cell section to be detected. To assess mitochondrial damage, cells were preincubated with $100 \mathrm{nM}$ TMRE (Invitrogen Life Technologies) or $3 \mu \mathrm{M}$ DHE (Invitrogen Life Technologies) for $30 \mathrm{~min}$ at $37^{\circ} \mathrm{C}$, and then brought to the microscope stage and equilibrated for a further $15-30 \mathrm{~min}$ before imaging. Cells were imaged using a $40 \times 10.85 \mathrm{NA}$ air objective and a 561-nm laser line.

To determine the kinetics of cell death, the elapsed time between NK-mediated granule delivery to cell rounding, and cell rounding to secondary necrosis were measured. To determine NK-mediated granule delivery, $100 \mu \mathrm{M} \mathrm{PI}$ was added to the culture immediately before imaging. Cell rounding was defined as the timepoint at which the target cell had shown defined cell contraction and/or detachment, as determined by progressive contrast around the cell membrane. Secondary necrosis was determined by staining with $1.5 \mu \mathrm{M} \mathrm{PI}$ in the culture.

All image analysis was performed using LAS AS version 1.7.0 (Leica Microsystems Gmb) and MetaMorph version 7.5.5.0 (MDS Analytical Technologies, Sunnyvale, CA, USA) image analysis software. Changes in intracellular fluorescence during the initiation phase of death were measured by drawing a region of interest around each dying cell to measure integrated intensity. Changes in fluorescence over time were expressed as relative change in integrated intensity, measured by dividing the fluorescence value of the target cell immediately before rounding by the fluorescence value of the target at an early initial reference timepoint, before engagement by any effector cells has occurred. To account for photobleaching over time, relative change in integrated intensity was also calculated for corresponding untreated targets, or targets that did not undergo NK attack in the vicinity of the dying cells of interest.

Immunofluorescence microscopy. Adherent target cells were plated overnight at $37^{\circ} \mathrm{C}$ with $10 \% \mathrm{CO}_{2}$ in ibiTreat eight-well chamber slides at a density of $1.5-3 \times 10^{4}$ cells per well. Following treatment to initiate cell death, the cells were immediately fixed to retain cell structure and stained with antibodies or fluorophores.

To visualise intracellular cytochrome $c$, cells were gently washed one to three times in cold PBS, and then fixed by incubating with $4 \%$ paraformaldehyde (Electron Microscopy Sciences, Hatfield, PA, USA) for 20 min. Excess paraformaldehyde was quenched with the addition of $50 \mathrm{mM}$ glycine for $20 \mathrm{~min}$, and then cells were incubated for $30 \mathrm{~min}$ in blocking/permeabilization buffer (PBS with 2\% BSA and $0.1 \%$ saponin). Cells were incubated with primary antibodies for $1 \mathrm{~h}$ at room temperature, then washed again and incubated with the appropriate fluorophoreconjugated secondary antibody for $1 \mathrm{~h}$ at room temperature. Cells were washed three times in PBS and stored at $4{ }^{\circ} \mathrm{C}$ in the dark until analysis. To visualise polymerised actin and microtubules, cells were fixed using a previously described protocol for the preservation of cytoskeletal elements. ${ }^{42,43}$ To visualise $\gamma$-tubulin staining, cells were fixed using a methanol fixation protocol, as previously described. ${ }^{44}$ Cells were stained with DAPI to visualise nuclei. Fluorescent cell staining was detected using a Leica TCS SP5 spectral confocal microscope with a $63 \times / 1.30$ NA glycerol objective.

Statistical analysis. All statistical analysis was performed using GraphPad Prism Version 5.0 (GraphPad Software Inc, San Diego, CA, USA). Error bars on all figures represent S.E.M. For timelapse experiments, the averages for each experiment were obtained by analysing many individual cells in each experiment. Data presented is the average of individual experiments \pm S.E.M. The total number of individual cells examined is also reported.

\section{Conflict of Interest}

The authors declare no conflict of interest
Acknowledgements. We thank Dr. Jenny Chia and Dr. Misty Jenkins with assistance in performing additional experiments, Mr. Kevin Thia for assistance in preparation of some of the figures and Dr. Jamie Lopez and Dr. Amelia Brennan for their helpful discussions. OS is supported by a Cancer Research Institute Pre-doctoral Tumour Immunology Scholarship. NJW is supported by a Futures Fellowship from the Australian Research Council (ARC) and the Prostate Cancer Foundation Australia. JAT and PIB are supported by Program and Project grants from the National Health and Medical Research Council (NHMRC), Australia.

\section{Author contributions}

OS designed and performed the experiments, analysed data and wrote the manuscript; SES performed experiments and produced recombinant Gzm A; IV, $\mathrm{DB}, \mathrm{MH}, \mathrm{SE}, \mathrm{SA}$ and KAS conducted additional experiments; PIB contributed intellectual input; NJW and JAT designed the experiments and wrote the manuscript.

1. Smyth MJ, Thia KY, Street SEA, MacGregor D, Godfrey DI, Trapani JA. Perforin-mediated cytotoxicity if critical for surveillance of spontaneous lymphoma. J Exp Med 2000; 192: 755-760.

2. Kagi D, Ledermann B, Burki K, Seiler P, Odermatt B, Olsen KJ et al. Cytotoxicity mediated by T cells and natural killer cells is greatly impaired in perforin-deficient mice. Nature 1994; 369: 31-37.

3. Trapani JA, Klein JL, White PC, Dupont B. Molecular cloning of an inducible serine esterase gene from human cytotoxic lymphocytes. Proc Natl Acad Sci USA 1988; 85: 6924-6928.

4. Beresford PJ, Xia Z, Greenberg AH, Lieberman J. Granzyme A loading induces rapid cytolysis and a novel form of dna damage independently of caspase activation. Immunity 1999; 10: 585-594.

5. Martinvalet D, Zhu P, Lieberman JL. Granzyme A induces caspase-independent mitochondrial damage, a required first step for apoptosis. Immunity 2005; 22: 355-370.

6. Martinvalet D, Dykxhoorn DM, Ferrini R, Lieberman J. Granzyme A cleaves a mitochondrial complex I protein to initiate caspase-independent cell death. Cell 2008; 133: 681-692.

7. Beresford PJ, Zhang D, Oh DY, Fan Z, Greer EL, Russo ML et al. Granzyme A activates an endoplasmic reticulum-associated caspase-independent nuclease to induce singlestranded DNA nicks. J Biol Chem 2001; 276: 43285-43293.

8. Metkar SS, Menaa C, Pardo J, Wang B, Wallich R, Freudenberg M et al. Human and Mouse Granzyme A Induce a Proinflammatory Cytokine Response. Immunity 2008; 29: 720-733.

9. Joeckel LT, Wallich R, Martin P, Sanchez-Martinez D, Weber FC, Martin SF et al. Mouse granzyme K has pro-inflammatory potential. Cell Death Differ 2011; 18: 1112-1119.

10. Kaiserman D, Bird CH, Sun J, Matthews A, Ung K, Whisstock JC et al. The major human and mouse granzymes are structurally and functionally divergent. J Cell Biol 2006; 175: $619-630$.

11. Shresta S, Russell JH, Ley TJ. Mechanisms responsible for Granzyme B-Independent Cytotoxicity. Blood 1997; 89: 4085-4091.

12. Pardo J, Bosque A, Brehm R, Wallich R, Naval J, Mullbacher A et al. Apoptotic pathways are selectively activated by granzyme A and/or granzyme B in CTL-mediated target cell lysis. J Cell Biol 2004; 167: 457-468.

13. Waterhouse NJ, Sutton VR, Sedelies KA, Ciccone A, Jenkins M, Turner SJ et al. Cytotoxic T lymphocyte-induced killing in the absence of granzymes $A$ and $B$ is unique and distinct from both apoptosis and perforin-dependent lysis. J Cell Biol 2006; 173: 133-144.

14. Yoshikawa Y, Hirayasu H, Tsuzuki S, Fushiki T. Granzyme A causes detachment of alveolar epithelial A549 cells accompanied by promotion of interleukin-8 release. Biosci Biotechnol Biochem 2008; 72: 2481-2484.

15. Simon MM, Kramer MD, Prester M, Gay S. Mouse T-cell associated serine proteinase 1 degrades collagen type IV: a structural basis for the migration of lymphocytes through vascular basement membranes. Immunology 1991; 73: 117-119.

16. Buzza MS, Bird PI. Extracellular granzymes: current perspectives. Biol Chem 2006; 387: 827-837.

17. Lopez JA, Susanto O, Jenkins MR, Lukoyanova N, Sutton VR, Law RH et al. Perforin forms transient pores on the target cell plasma membrane to facilitate rapid access of granzymes during killer cell attack. Blood 2013; 121: 2659-2668.

18. Sutton VR, Wowk ME, Cancilla M, Trapani JA. Caspase activation by granzyme B is indirect, and caspase autoprocessing requires the release of proapoptotic mitochondrial factors. Immunity 2003; 18: 319-329.

19. Sebbagh M, Hamelin J, Bertoglio J, Solary E, Breard J. Direct cleavage of ROCK II by granzyme $B$ induces target cell membrane blebbing in a caspase-independent manner. J Exp Med 2005; 201: 465-471.

20. Degterev A, Hitomi J, Germscheid M, Ch'en IL, Korkina O, Teng X et al. Identification of RIP1 kinase as a specific cellular target of necrostatins. Nat Chem Biol 2008; 4: 313-321.

21. Van Damme P, Maurer-Stroh S, Hao H, Colaert N, Timmerman E, Eisenhabler F et al. The substrate specificity profile of human granzyme A. Biol Chem 2010; 391: 983-997.

22. Luders J, Stearns T. Microtubule-organizing centres: a re-evaluation. Nat Rev Mol Cell Biol 2007; 8: 161-167. 
23. Bornens M. Is the centriole bound to the nuclear membrane? Nature 1977; $270: 80-82$.

24. Anthony DA, Andrews DM, Chow M, Watt SV, House C, Akira S et al. A role for granzyme $M$ in TLR4-driven inflammation and endotoxicosis. J Immunol 2010; 185: 1794-1803.

25. Fan Z, Beresford PJ, Zhang D, Xu Z, Novina CD, Yoshida A et al. Cleaving the oxidative repair protein Ape1 enhances cell death mediated by granzyme A. Nat Immunol 2003; 4 145-153.

26. Gourlay CW, Carpp LN, Timpson P, Winder SJ, Ayscough KR. A role for the actin cytoskeleton in cell death and aging in yeast. J Cell Biol 2004; 164: 803-809.

27. Wilson CA, Tsuchida MA, Allen GM, Barnhardt EL, Applegate KT, Yam PT et al. Myosin I contributes to cell-scale actin network treadmilling through network disassembly. Nature 2010; 465: 373-379.

28. Shresta S, Maclvor DM, Heusel JW, Russell JH, Ley TJ. Natural killer and lymphokineactivated killer cells require granzyme $B$ for the rapid induction of apoptosis in susceptible target cells. Proc Natl Acad Sci USA 1995; 92: 5679-5683.

29. Mullbacher A, Waring P, Hla RT, Tran T, Chin S, Stehle T et al. Granzymes are the essential downstream effector molecules for the control of primary virus infections by cytolytic leukocytes. Proc Natl Acad Sci USA 1999; 96: 13950-13955.

30. Sedelies KA, Ciccone A, Clarke CJP, Oliaro J, Sutton VR, Scott FL et al. Blocking granulemediated death by primary human NK cells requires both protection of mitochondria and inhibition of caspase activity. Cell Death Differ 2008; 15: 708-717.

31. Pegram HJ, Haynes NM, Smyth MJ, Kershaw MH, Darcy PK. Characterizing the anti-tumor function of adoptively transferred NK cells in vivo. Cancer Immunol Immunother 2010; 59: 1235-1246.

32. Ebnet K, Hausmann M, Lehmann-Grube F, Mullbacher A, Kopf M, Lamers M et al. Granzyme A-deficient mice retain potent cell-mediated cytotoxicity. EMBO J 1995; 14 4230-4239.

33. Heusel JW, Wesselschmidt RL, Shresta S, Russell JH, Ley TJ. Cytotoxic lymphocytes require granzyme $B$ for the rapid induction of DNA fragmentation and apoptosis in allogeneic target cells. Cell 1994; 76: 977-987.

34. Simon MM, Hausmann M, Tran T, Ebnet K, Tschopp J, ThaHla R et al. In vitro- and ex vivo-derived cytolytic leukocytes from granzyme $\mathrm{a} \times \mathrm{b}$ double knockout mice are defective in granule-mediated apoptosis but not lysis of target cells. J Exp Med 1997; 186: $1781-1786$

35. Kagi D, Ledermann B, Burki K, Hengartner H, Zinkernagel RM. CD8 + T cell-mediated protection against an intracellular bacterium by perforin-dependent cytotoxicity. Eur J Immunol 1994; 24: 3068-3072.

36. Sun J, Bird CH, Buzza MS, McKee KE, Whisstock JC, Bird PI. Expression and purification of recombinant human granzyme B from Pichia pastoris. Biochem Biophys Res Commun 1999; 261: 251-255.

37. Sun J, Bird CH, Thia KY, Matthews AY, Trapani JA, Bird PI. Granzyme B encoded by the commonly ocurring human RAH allele retains pro-apoptotic activity. J Biol Chem 2004; 279: 16907-16911.

38. Sutton VR, Waterhouse NJ, Baran K, Browne KA, Voskoboinik I, Trapani JA. Measuring cell death mediated by cytotoxic lymphocytes or their granule effector molecules. Methods 2008; 44: 241-249.

39. Bird CH, Sun J, Ung K, Karambalis D, Whisstock JC, Trapani JA et al. Cationic sites on granyzme $\mathrm{B}$ contribute to cytotoxicity by promoting its uptake into target cells. Mol Cell Biol 2005; 25: 7854-7867.

40. Voskoboinik I, Thia MC, Fletcher J, Ciccone A, Browne KA, Smyth MJ et al. Calciumdependent plasma membrane binding and cell lysis by perforin are mediated through its $\mathrm{C} 2$ domain: a critical role for aspartate residues $429,435,483$, and 485 but not 491 . J Biol Chem 2005; 280: 8426-8434.

41. Voskoboinik I, Thia MC, De Bono A, Browne KA, Cretney E, Jackson JT et al. The functional basis for hemophagocytic lymphohistiocytosis in a patient with co-inherited missense mutations in the perforin (PFN1) gene. J Exp Med 2004; 200: 811-816.

42. Schuh M. An actin-dependent mechanism for long-range vesicle transport. Nat Cell Biol 2011; 13: 1431-1436

43. Strickland L, von Dassow G, Ellenberg J, Foe V, Lenart P, Burgess D. Light microscopy of echinoderm embryos. Methods Cell Biol 2004; 74: 371-409.

44. Jenkins MR, Tsun A, Stinchcombe JC, Griffiths GM. The strength of T cell receptor signal controls the polarization of cytotoxic machinery to the immunological synapse. Immunity 2009; 31: 621-631.

Supplementary Information accompanies this paper on Cell Death and Differentiation website (http://www.nature.com/cdd) 